\title{
Meson Form Factors and Deep Exclusive Meson Production Experiments
}

\author{
Tanja Horn ${ }^{1,2, a}$ \\ ${ }^{1}$ Catholic University of America, Washington, DC 20064, USA \\ ${ }^{2}$ Jefferson Laboratory, Newport News, VA 23606, USA
}

\begin{abstract}
Pion and kaon electroproduction data play a unique role in Nature and our understanding of them is essential for explaining hadron structure. Precision longitudinaltransverse separated pion and kaon cross sections are of particular interest. They allow for the extraction of meson form factors and validation of understanding of hard exclusive and semi-inclusive reactions $\left(\pi^{+}, K^{+}, \pi^{0}, \gamma\right)$ towards 3D hadron imaging and potential future flavor decomposition. We review recent data and present prospects for deep exclusive pion and kaon electroproduction at the $12 \mathrm{GeV}$ Jefferson Lab including the prospects to use projected charged- and neutral pion data to further determine the spin, charge-parity and flavor of GPDs, including the helicity-flip GPDs.
\end{abstract}

\section{Introduction}

The pion is the lightest quark system, with a single valence quark and a single valence antiquark. It is also the particle responsible for the long range character of the strong interaction that binds the atomic nucleus together. A general belief is that the rules governing the strong interaction are left-right, i.e. chirally, symmetric. If this were true, the pion would have no mass. The chiral symmetry of massless Quantum Chromodynamics (QCD) is broken dynamically by quark-gluon interactions and explicitly by inclusion of light quark masses, giving the pion and kaon mass [1,2]. The pion and kaon are thus seen as the key to confirm the mechanism that dynamically generates nearly all of the mass of hadrons and central to the effort to understand hadron structure [3].

Prominent examples of experimental observables that provide basic information about pion and kaon structure are the form factors [3-13]. The last decade has seen a dramatic improvement in the understanding of pion electroproduction data [14-19], which has given confidence in the reliability of the electroproduction method yielding the physical pion form factor. In general, the extraction of precision meson form factors from experiment requires a longitudinal-transverse $(\mathrm{L} / \mathrm{T})$ separation of the cross section isolating $\sigma_{L}$, proper selection of the pion pole process, extraction of the form factor using a model, and the validation of the technique.

Recent calculations [3] show that one may expect dominance of hard contributions to the pion form factor for $Q^{2} \sim 8 \mathrm{GeV}^{2}$ [20]. There, it is predicted that $F_{\pi}$ will start exhibiting precisely the momentum-dependence from QCD, a power law behavior plus logarithmic corrections to scaling, but with the normalization fixed by a pion wave function whose dilation with respect to the asymptotic

\footnotetext{
ae-mail: hornt@cua.edu
} 
form is a definite signature of Dynamical Chiral Symmetry Breaking (DCSB). Data extending into this regime would thus for the first time have the potential to quantitatively reveal hard QCDs signatures.

Key properties of the nucleon are encoded in the correlation between their longitudinal momentum and their transverse position, such as the orbital angular momentum. This correlation is expressed through Generalized Parton Distributions (GPDs) [21-24], which can be understood as spatial densities at different values of the longitudinal momentum of the quark, and due to the space-momentum correlation information encoded in the GPDs can link through the Ji sum rule to a parton's angular momentum [25].

The key to extracting GPDs from experiment are QCD factorization theorems [26, 27], which allow the amplitudes for deep exclusive processes to be expressed in terms of GPDs [22, 25, 28]. GPD measurements at Jefferson Lab rely on the assumption that deep exclusive reactions are well described by their leading twist mechanism. The value of $Q^{2}$ at which this approximation is valid experimentally needs to be determined and the contributions of higher twist components to observables need to be quantified. The $Q^{2}$ dependence of cross sections is the only unambiguous way to separate higher twist contributions to exclusive channels.

Exclusive meson production provides observables that allow for separating different quark and anti-quark flavors [23]. The theoretical description of these processes is more complicated than exclusive photon production, and thus measurements that provide information about the reaction mechanism, e.g., tests of hard-soft factorization, are essential. In particular, the emerging transversity GPDs [29-31] may be accessed if dominance of the transverse cross section at small values of $t<0.3$ $\mathrm{GeV}^{2}$ can be experimentally verified.

To validate the meson factorization theorems and potentially extract flavor separated GPDs from experiment, one has to measure the longitudinal-transverse (L/T) separated cross sections and their $t$ and $Q^{2}$ dependencies. Only $\mathrm{L} / \mathrm{T}$ separated cross sections can unambigously show the dominance of longitudinal or transverse photons and allow one to determine possible correlations in $t$ and $Q^{2}$. The onset of factorization for light mesons may be expected earlier than for heavier ones. Thus, if meson factorization is to be observed it is most probable for pion and kaon.

Measuring L/T separated cross sections places strong demands on experimental facilities requireing rigorous control over systematic uncertainties. Hall $\mathrm{C}$ at the Jefferson Laboratory (JLab) with its precision focusing spectrometers and particle identification detectors is the only facility available for carrying out these measurements. Two experiments are planned with the $12 \mathrm{GeV} \mathrm{JLab}$ extending charged pion data to $Q^{2}$ of about $9 \mathrm{GeV}^{2}$ [32] and kaon data to $5.5 \mathrm{GeV}^{2}$ [33].

\section{Experimental Determination of Pion and Kaon form factors}

At low values of $Q^{2}, F_{\pi}$ has been determined directly up to photon energies of $Q^{2}=0.253 \mathrm{GeV}^{2}$ at Fermilab [34] and at the CERN SPS [8] from the scattering of high-energy, charged pions by atomic electrons. These data were used to constrain the charge radius of the pion, which is determined to be $r_{\pi}=0.657 \pm 0.012 \mathrm{fm}$. Due to kinematic limitation in the energy of the pion beam and unfavorable momentum transfer one has to resort to other experimental methods to reach the higher $Q^{2}$ regime. At higher values of $Q^{2} F_{\pi}$ can be determined from electroproduction of pions on the proton.

In electroproduction experiments one detects the scattered electron and the electroproduced charged pion (or kaon) in coincidence. The differential cross section can be expressed in terms of a known electrodynamic factor and a virtual photoproduction cross section. The latter can be expressed in terms of the linear combinations of the products of virtual-photoproduction helicity amplitudes, which are the unpolarized transverse production, the purely scalar (longitudinal) production, and the interference terms between the transverse and transverse-scalar states. This reduced cross section can 
be written as a sum of four separate cross sections or structure functions, which depend on $W, Q^{2}$ and $t$

$$
2 \pi \frac{d^{2} \sigma}{d t d \phi}=\frac{d \sigma_{T}}{d t}+\epsilon \frac{d \sigma_{L}}{d t}+\sqrt{2 \epsilon(1+\epsilon)} \frac{d \sigma_{L T}}{d t} \cos \phi+\epsilon \frac{d \sigma_{T T}}{d t} \cos 2 \phi
$$

Here, $\epsilon=\left(1+\frac{2|\mathbf{q}|^{2}}{Q^{2}} \tan ^{2} \frac{\theta_{e}}{2}\right)^{-1}$ is the polarization of the virtual photon with $\mathbf{q}$ denoting the threemomentum of the transferred virtual photon and $\theta_{e}$ the electron scattering angle, and $\phi$ is the angle between the scattering plane defined by the incoming and scattered electrons and the reaction plane defined by the transferred virtual photon and the scattered meson. To separate the different structure functions one has to determine the cross section for at least two sufficiently different values of $\epsilon$ as a function of the angle $\phi$ for fixed values of the invariant mass of the virtual photon-nucleon system, $W$, $Q^{2}$ and the Mandelstam variable, $t$. The dominance of the pion pole in the longitudinal cross section, $\sigma_{L}$, and its characteristic $t$ dependence allow for extractions of electromagnetic form factors from these data.

Cross section data suggest a dominant pion pole in the longitudinal $\pi^{+}$cross section at values of $-t<0.3(\mathrm{GeV} / \mathrm{c})^{2}$ [35]. The observation of a dominant pion pole alone is, however, not sufficient to make a precise extraction of $F_{\pi}$ from experiment. To minimize background contributions, the longitudinal cross section $\sigma_{L}$, is thus isolated via a Rosenbluth $\mathrm{L} / \mathrm{T}(\mathrm{LT} / \mathrm{TT})$ separation. Without an explicit $\mathrm{L} / \mathrm{T}$ separation it is not clear what fraction of the cross section is due to longitudinal photons and what the contribution of the pole to it is in these kinematics.

With the availability of high-intensity electron beams, combined with accurate magnetic spectrometers at JLab, it has been possible to determine L/T separated cross sections with high precision. The measurement of these cross sections in the regime of $Q^{2}=0.60-1.60 \mathrm{GeV}^{2}$ (Experiment Fpi-1 [36, 37]) and $Q^{2}=1.60-2.45 \mathrm{GeV}^{2}$ (Experiment Fpi-2 [14] and pionCT [15]) are described in detail in Ref. [38]. Pion electroproduction experiments are performed at the smallest possible value of $t$, which is still a distance away from the pion pole. The pion form factor thus has to be obtained either by kinematic extrapolation or by using a theoretical model. In this method consistency between data and model is essential.

Experiments like those discussed in Refs. [14-16, 38, 39] have used a Regge model for pion electroproduction developed by Vanderhaeghen, Guidal and Laget (VGL) $[39,40]$ for the extraction of $F_{\pi}$. In this model the pole-like propagators of Born term models are replaced with Regge propagators, i.e., the interaction is effectively described by the exchange of a family of particles with the same quantum numbers instead of a single particle. The model parameters are fixed from pion photoproduction data. The free parameters are $\Lambda_{\pi}$ and $\Lambda_{\rho}$, the trajectory cutoffs. A fit to the model longitudinal cross section then gives $F_{\pi}$ at each value of $Q^{2}$.

To give confidence in the electroproduction method yielding the physical form factor, one can carry out several experimental studies. These include checking the consistency of the model with the data, extracting the form factor at several values of $t_{\min }$ for fixed $Q^{2}$, verifying that the pole diagram is the dominant contribution to the reaction mechanism, and verifying that the electroproduction technique gives results consistent with those from $\pi-e$ elastic scattering at the same $Q^{2}$. In Ref. [16], to check if the VGL Regge model properly accounts for the pion production mechanism, spectator nucleon, and other off-shell ( $t$ dependent) effects, $F_{\pi}$ was extracted at different distances in $t$ from the pion pole. The resulting $F_{\pi}$ values agree to $4 \%$ and did not depend on the $t$ acceptance, which lends confidence in the applicability of the VGL model to the kinematic regime of the data and the validity of the extracted $F_{\pi}$ values. The dominance of the $t$-channel process in $\sigma_{L}$ was verified in Ref. [18, 19] through the charged pion longitudinal cross section ratios, $R_{L}=\sigma_{L}\left[n\left(e, e \prime \pi^{-}\right) p\right] / \sigma_{L}\left[p\left(e, e \prime \pi^{+}\right) n\right]$, obtained with a deuterium target. The $t$-channel diagram is purely isovector and so any isoscalar back- 
ground contributions like $b_{1}(1235)$ to the $t$ channel will dilute the ratio. With increasing $t$, the transverse cross section ratio is expected to approach the ratio of quark charges, i.e., $1 / 4$. The data show that $R_{L}$ approaches the pion charge ratio, consistent with pion-pole dominance. The $t$-dependence of the transverse cross section ratio, $R_{T}=\sigma_{T}\left[n\left(e, e \imath \pi^{-}\right) p\right] / \sigma_{T}\left[p\left(e, e l \pi^{+}\right) n\right]$, shows a rapid fall-off consistent with $s$-channel quark knockout. Direct comparison of $F_{\pi}$ values extracted from very low $t$ electroproduction with the exact values measured in elastic $\pi-e$ scattering showed that the data are consistent within the uncertainties lending further confidence in the validity of the extracted $F_{\pi}$ values.

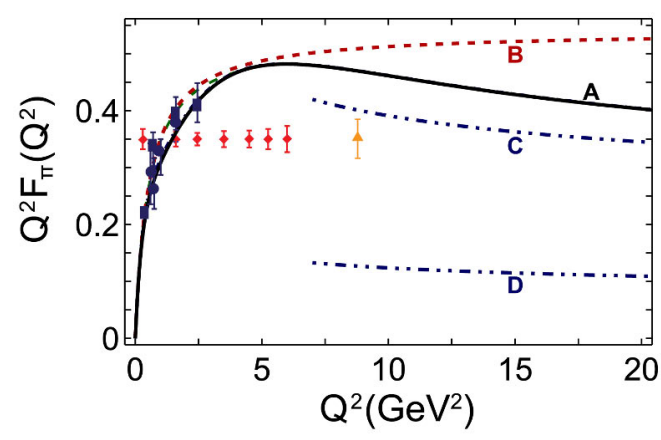

Figure 1. Existing and projected data on the pion form factor. The curves are discussed in the text.

The world precision $F_{\pi}$ data are summarized by the blue points in Fig. 1. The orange point is the projected high- $Q^{2}$ point for $12 \mathrm{GeV}$ JLab [32] taking into account recent experimental progress. The dotted curve $(\mathrm{B})$ is the maximum-allowed value of the form factor as set by the pion radius. The dot-dot-dashed curves illustrate the calculations from short-distance view assuming either a point-like asymptotic wave function of the pion (D) or a modern wave function (C). The solid curve (A) is the QCD-theory prediction bridging these long- and short-distance domains.

As with elastic scattering, these data allow for extracting the pion charge radius as has been done in Ref. [41, 42] for spacelike and in Refs. [43] for timelike conditions. In the latter the extension to spacelike domain is accomplished by the use of dispersion relations and models to obtain separate real and imaginary parts. In Ref. [41] the use of models is avoided and the impact of new experiments evaluated. This first attempt of a detailed analysis with spacelike data shows that the transverse charge density of the pion is larger than that of the proton close to the core and that the two densities coalesce for impact parameters greater than $0.3 \mathrm{fm}$. The former is expected since the pion radius is smaller than that of the proton. The latter may be interpreted as the proton consisting of a non-chiral core occupying most of the volume and a meson cloud at large impact parameters.

The next simplest meson available for experimental studies and also containing strangeness is the kaon. Of particular interest to hadron structure is the effect of the heavier strange quark in the kaon [44]. Similar to pions the kaon form factor has been determined directly up to photon energies of $Q^{2}=0.10 \mathrm{GeV}^{2}$ at Fermilab [45] and at the CERN SPS [46] from the scattering of high-energy, charged kaons by atomic electrons. These data were used to constrain the mean square charge radius of the kaon, which is determined to be $<r_{K}^{2}>=0.34 \pm 0.05 \mathrm{fm}^{2}$. In general, the kaon charge radius is expected to be smaller than that of the pion due to the presence of the heavier strange quark. At higher energies the kaon form factor can, in principle, be extracted from kaon electroproduction data. However, there are experimental challenges that have to be addressed. In particular, the kaon 
pole is farther from the physical region than the pion, which may raise doubts about the ability to obtain reliable information on $F_{K}$ from kaon electroproduction data. Two experimental points are important to consider in this respect. First, extraction of the pion form factor from similar pion electroproduction data at small $t$ is completed by carefully studying the model dependence of the analysis, not by direct extrapolation; and this justifies greater confidence in this method. Second, initial comparative extractions of the pion form factor from low- $t$ and larger- $t$ data suggest only a modest model dependence; and thelarger- $t$ pion data lie at a similar distance from the pole as most of the projected kaon data discussed herein. It is thus reasonable to imagine that $F_{K}$ may be extracted from kaon electroproduction data in a similar fashion, albeit with a larger model dependence than $F_{\pi}$.

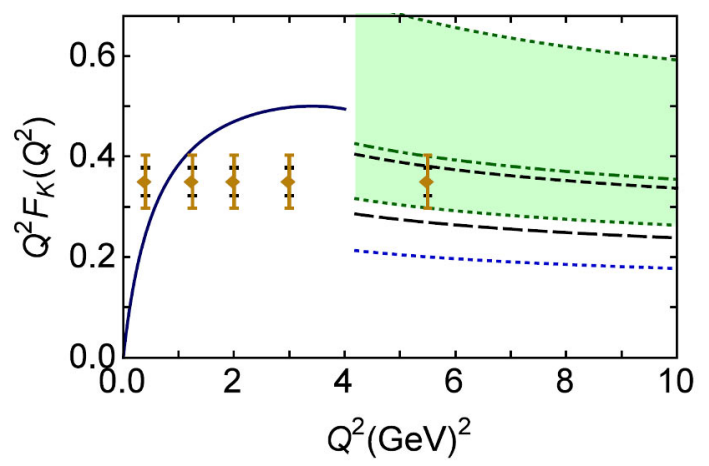

Figure 2. Projected reach for kaon form factor anticipated from a $12 \mathrm{GeV} \mathrm{JLab} \mathrm{experiment.} \mathrm{The} \mathrm{error} \mathrm{estimates}$ are based on different assumptions about the $t$ - and model-dependence of the form factor extractions, with the larger uncertainty being conservative. The curves are discussed in the text.

Nevertheless, detailed demonstration of the dominance of the kaon pole is required if one is to be confident in the extraction of $F_{K}$. Current electroproduction data show that the $t$-dependence of the longitudinal kaon cross section is less steep than that of the pion $[17,35]$. These data include $\mathrm{L} / \mathrm{T}$ separated cross sections up to photon energies of $Q^{2}=2.35 \mathrm{GeV}^{2}$ from Refs. [47, 48]. Additional kaon electroproduction data from Jefferson Lab are under analysis. The results from Refs.[17, 35] are consistent with the kaon pole factor, $-t /\left(t-m_{K}^{2}\right)^{2}$, giving less enhancement than that of the pion. However, calculations predict a small maximum in the kaon cross section near $t=0.1 \mathrm{GeV}^{2}$ due to the kaon pole $[29,30]$.

Access to small values of $t$ and $\mathrm{L} / \mathrm{T}$ separations of the kaon cross section will be enabled by the $12 \mathrm{GeV}$ Jefferson Lab. The projected reach and accuracy anticipated are shown in Fig. 2. The curves depict theoretical calculations obtained from the hard-scattering formula when different kaon pion distribution amplitudes (PDAs) are used: long-dashed (black) curve, DSE prediction; dot-dashed (green) curve, inferred from lattice QCD values of the lowest two moments, with the green band indicating the uncertainty; dashed (black) curve, obtained from the long-dashed curve by supposing that the second moment of the PDA is just 10\% larger; and dotted (blue) curve, the conformal-limit PDA. The solid (blue) curve is the prediction from Ref. [49]. Further details can be found in Ref. [2]

Current experiments have established the techniques for meson electroproduction experiments and the determination of the meson form factors from these data. The $12 \mathrm{GeV}$ upgrade at the Jefferson Lab features new instrumentation that allows for pushing precision meson form factor measurements to the highest momentum transfers to date. Planned experiments aim for precision measurements of 
the pion form factor to $Q^{2}=6 \mathrm{GeV}^{2}$ and also have the potential to determine the pion form factor up to $Q^{2} \sim 9 \mathrm{GeV}^{2}$ [32]. These measurements are made possible by the combination of the two moderate acceptance, magnetic spectrometers in Hall C. The "High Momentum Spectrometer" (HMS) provides angular acceptance of $6 \mathrm{msr}$ and can detect particles with momenta up to $7 \mathrm{GeV} / \mathrm{c}$. The new "SuperHigh Momentum Spectrometer" (SHMS) features a solid angle of about $4 \mathrm{msr}$, a momentum coverage up to $11 \mathrm{GeV} / \mathrm{c}$, and covers scattering angles between 5.5 and 40 degrees. The small scattering angle capability combined with excellent control of systematic uncertainties, kinematic reproducibility and well-understood acceptance allows for measurements of precision cross sections and L/T separations at high luminosity $\left(>10^{38} / \mathrm{cm}^{2} \mathrm{~s}\right.$ ). Such data allow for extractions of the pion form factor at nearly quadruple the momentum transfer over which it is currently known. QCD backgrounds in these data at high values of $Q^{2}$ and $t$ can be addressed experimentally through measurements of the charged pion ratio as discussed above or of the neutral pion cross section. The charged pion $t$-channel diagram is purely isovector. Isaoscalar backgrounds would distort the ratio. The neutral pion production reaction does not have a large pion pole contribution. Measurements of the longitudinal cross section for exclusive neutral pion production like those in Ref. [50] should thus be sensitive to non-pole contributions in the charged pion cross section.

The small-angle capability of the magnetic spectrometers and availability of electron beam energies of $11 \mathrm{GeV}$ will also allow for measurements of the $\mathrm{L} / \mathrm{T}$ separated kaon cross section at the highest values of $Q^{2}$ to date [33]. The challenge in extracting the kaon form factor from cross section data is that one has to demonstrate that the kaon pole dominates. This can be done in a similar way as for the pion. In addition, the dominance of the kaon pole can, in principle, be tested through the ratio of the longitudinal cross sections of $\sigma^{0}$ to $\Lambda$ channels. The SHMS and HMS missing mass resolution is expected to be very good and together with the spectrometer coincidence acceptance will allow for simultaneous measurements of the $\Lambda$ and $\Sigma^{0}$ channels. If the kaon pole dominates the ratio should be similar to the ratio of the coupling constants $g_{p K \Lambda}^{2} / g_{p K \Sigma}^{2}$.

\section{Validation of the exclusive reaction mechanism}

CEBAF, the electron accelerator at JLab, has become the world's most advanced particle accelerator for investigating the nucleus of the atom, the protons and neutrons making up the nucleus, and the quarks and gluons inside them. The $12-\mathrm{GeV}$ beam will soon allow revolutionary access to a new representation of the proton's inner structure. In the past, our knowledge has been limited to one-dimensional spatial densities (form factors) and longitudinal momentum densities (parton distributions). This cannot describe the proton's true inner structure, as it will, for instance, be impossible to describe orbital angular momentum, an important aspect for nucleon spin, for which one needs to describe the correlation between the momentum and spatial coordinates.

A three-dimensional description of the nucleon has been developed through the Generalized Parton Distributions (GPDs) [21-24] and the Transverse Momentum-Dependent parton distributions (TMDs) [51]. GPDs can be viewed as spatial densities at different values of the longitudinal momentum of the quark, and due to the space-momentum correlation information encoded in the GPDs, can link through the so-called Ji sum rule to a parton's angular momentum [25]. The TMDs are functions of both the longitudinal and transverse momentum of partons, and they offer a momentum tomography of the nucleon complementary to the spatial tomography of GPDs. The GPD and TMD programs are at the heart of the scientific motivation of the $12 \mathrm{GeV}$ Jefferson Lab [52].

To extract the rich information on proton structure encoded in the GPD and TMD frameworks, it is of prime importance to show in accurate measurements, pushing the energy scales, that the scattering process is understood. In perturbative QCD at leading twist, there are eight GPDs (four helicityconserving distributions $H, E, \tilde{H}, \tilde{E}$ and four helicity flip ones $H^{T}, E_{T}, \tilde{H}_{T}, \tilde{E}_{T}$ ), which represent 
the various helicity-spin transitions between the initial and final states of the quark-nucleon system. The value of $Q^{2}$ at which this the leading twist approximation is valid needs to be experimentally determined and the contributions of higher twist components to observables need to be quantified. The $Q^{2}$ dependence of $\mathrm{L} / \mathrm{T}$ separated cross sections is the only unambiguous way to separate higher twist contributions to exclusive channels.

JLab $12 \mathrm{GeV}$ features two experiments emphasizing kaon and pion L/T separated cross sections to the highest values of $Q^{2}$ achievable with this facility. E12-09-011 [33] will provide the first $\mathrm{L} / \mathrm{T}$ separated kaon cross sections beyond the resonance region to $Q^{2}=5.5 \mathrm{GeV}^{2}$. This will for the first time allow tests of the reaction mechanism in the transition from hadronic to partonic degrees of freedom when strangeness is in play. The data may also allow extractions of the kaon form factor at lower values of $t$ than previously accessible. The anticipated results will provide the most precise spatial mapping of the kaon to date and may reveal new insight on the role of the strange quark. E12-07-105 [32] aims primarily to probe conditions for factorization of deep exclusive measurements for charged pions (the simplest reaction beyond DVCS). E12-07-105 will provide L/T separated pion cross sections up to $Q^{2}=9 \mathrm{GeV}^{2}$, which can be used to verify the applicability of the GPD formalism and to constrain theoretical models. This experiment will also extend pion form factor data up to the highest possible momentum transfers achievable at a $12 \mathrm{GeV}$ Jefferson Lab $-Q^{2}=8.5 \mathrm{GeV}^{2}$. These data would allow for confirming recent calculations tied to dynamical chiral symmetry breaking, and thus could contribute a major step forward towards our understanding of QCD.

\section{Summary}

Meson form factor measurements play an important role in our understanding of hadron structure and their interactions. JLab $12 \mathrm{GeV}$ will dramatically improve the pion and kaon electroproduction data set making possible the extraction of meson form factors and the interpretation of the $12 \mathrm{GeV}$ JLab GPD data. Precision fully L/T separated pion and kaon cross section measurements will allow for accessing flavor separated GPDs. If confirmed experimentally, a large contribution of transverse photons would allow accessing a new class of GPDs. L/T separated cross sections will also make possible understanding of dynamical effects in both $Q^{2}$ and $-t$, which are important for the interpretation of non-perturbative contributions in experimentally accessible kinematics. Beyond $12 \mathrm{GeV} \mathrm{JLab,}$ the planned Electron-Ion Collider provides interesting opportunities to map pion and kaon structure functions over a wide kinematic range. The structure functions in terms of sea quarks and gluons are expected to be different for pions, kaons, and nucleons and to provide basic information about their origin of mass.

\section{References}

[1] C.D. Roberts, Perspective on the origin of hadron masses, arXiv:1606.03909 (2016).

[2] T. Horn and C.D. Roberts, J. Phys. 43 (2016) no. 7, 073001.

[3] L. Chang, I.C. Cloet, J.J. Cobos-Martinez, C.D. Roberts, S.M. Schmidt, P.C. Tandy, Phys. Rev. Lett. 110 (2013) 132001.

[4] W.R. Frazer, Phys. Rev. 115 (1959) 1763.

[5] G.R. Farrar, D.R. Jackson, Phys. Rev. Lett. 43 (1979) 246.

[6] A.V. Efremov, A.V. Radyushkin, Phys. Lett. B94 (1980) 245.

[7] V.A. Nesterenko, A.V. Radyushkin, Phys. Lett. B115 (1982) 410.

[8] S.R. Amendolia et al., Nucl. Phys. B277 (1986) 168; Phys. Lett. B146 (1984) 116.

[9] A.P. Bakulev, A.V. Radyushkin, Phys. Lett. B271 (1991) 223. 
[10] R. Jacob, P. Kroll, Phys. Lett. B 315 (1993) 463.

[11] C.D. Roberts, Nucl. Phys. A 605 (1996) 475.

[12] H.B. O’Connell, B.C. Pearce, A.W. Thomas, A.G. Williams, Prog Part. Nucl. Phys 39 (1997) 201.

[13] S. Brodsky, G. deTeramond, Phys. Rev. D 77 (2008) 056007.

[14] T. Horn et al., Phys.Rev.Lett. 97 (2006) 192001.

[15] T. Horn, X. Qian, et al., Phys. Rev. C 78, 058201 (2008).

[16] G. M. Huber, H. P. Blok, T. Horn, et al., Phys. Rev. C 78, 045203 (2008).

[17] T. Horn, Phys. Rev. C 85, 018202 (2012).

[18] G.M. Huber, H.P. Blok, C. Butuceanu, D. Gaskell, T. Horn, D.J. Mack, et al., Phys. Rev. Lett. 112 (2014) 18, 182501.

[19] G.M. Huber, H.P. Blok, C. Butuceanu, D. Gaskell, T. Horn, D.J. Mack, et al., Phys. Rev. C 91 (2015) 18, 182501.

[20] L. Chang, I.C. Cloet, C.D. Roberts, S.M. Schmidt, P.C. Tandy, Phys. Rev. Lett. 111 (2013) 141802.

[21] M. Diehl, Eur. Phys. J. C 25, 233 (2002); Phys. Rept. 388, 41 (2003).

[22] A.V. Radyushkin, Phys. Lett. B 385, 333 (1996); Phys. Lett. B 380, 417 (1996).

[23] K. Goeke, M.V. Polyakov and M. Vanderhaeghen, Prog. Part. Nucl. Phys. 47, 401 (2001)

[24] A.V. Belitsky and A.V. Radyushkin, Phys. Rept. 418, 1 (2005).

[25] X. Ji, Phys. Rev. D 55, 7114 (1997); Phys. Rev. Lett.78, 610 (1997).

[26] J.C. Collins, L. Frankfurt, M. Strikman, Phys. Rev. D 56, 2982 (1997).

[27] J.C. Collins, A. Freund, Phys. Rev. D 59, 074009 (1999).

[28] D. Mueller, D. Robaschik, B. Geyer, F.M. Dittes, J. Horejsi, Fortschr. Phys. 42, 101 (1994).

[29] S. V. Goloskokov and P. Kroll, Eur. Phys. J. C 65, 137 (2010) [arXiv:0906.0460 [hep-ph]].

[30] S. V. Goloskokov and P. Kroll, Eur. Phys. J. A 47, 112 (2011) [arXiv:1106.4897 [hep-ph]].

[31] G. R. Goldstein, J. O. Gonzales, Hernandez, S. Liuti, J. Phys. G 39 (2012) 115001.

[32] T. Horn, G. Huber, et al.,Approved JLab experiment E12-07-105: Scaling Study of the L-T Separated Pion Electroproduction Cross Section at $11 \mathrm{GeV}$.

Available online: http://www.jlab.org/exp_prog/proposals/07/PR12-07-103.pdf

[33] T. Horn, G. Huber, P. Markowitz et al., Approved JLab experiment E12-09-011: Studies of the L-T Separated Kaon Electroproduction Cross Section from 5-11 GeV.

Available online: http://www.jlab.org/exp_prog/proposals/09/PR12-09-011.pdf

[34] E.B. Dally et al., Phys. Rev. Lett.48 (1982) 375; Phys. Rev. D 24 (1981) 1718.

[35] L. Favart, T. Horn, M. Guidal, P. Kroll, Eur. J. Phys. A 52 (2016) no.6, 158.

[36] J. Volmer, et al., Phys. Rev. Lett. 86, (2000) 1713

[37] V. Tadevosyan, et al., Phys. Rev. C 75, (2007) 055205

[38] H. P. Blok, T. Horn, et al., Phys. Rev. C 78, 045202 (2008)

[39] M. Guidal, J-M Laget, M. Vanderhaeghen, Nucl. Phys. A 627, (1997) 645

[40] M. Vanderhaeghen, M. Guidal, J-M Laget, Phys. Rev. C 57, (1998) 1454

[41] M.A.P. Carmignotto, T. Horn, G. A. Miller, Phys. Rev. C 90 (2014) 025211.

[42] G. A. Miller, Phys. Rev. C 79 (2009) 055204.

[43] G. A. Miller, M. Strikman, C. Weiss, Phys. Rev. C 83 (2011) 015203; Phys. Rev. D 83 (2011) 013006.

[44] P.T.P Hutauruk, I.C. Cloet, A.W. Thomas, Phys. Rev. C 94 (2016) no.3, 035201. 
[45] E.B. Dally et al., Phys. Rev. Lett. 45 (1980) 232.

[46] S.R. Amendolia et al., Phys. Lett. B178 (1986) 435.

[47] R.M. Mohring et al., Phys. Rev. C 67 (2003) 055205.

[48] M. Coman et al., Phys. Rev. C 81 (2010) 052201.

[49] P. Maris, P. Tandy, Phys. Rev. C 62 (2000) 055204.

[50] C. Munoz-Camacho et al. and the Neutral Particle Spectrometer Collaboration, Approved Jefferson Lab experiment E12-13-010: Exclusive Deeply Virtual Compton and Neutral Pion CrossSection Measurements in Hall C.

Available online: https://www.jlab.org/exp_prog/proposals/13/PR12-13-010.pdf

[51] M Burkardt, Nucl. Phys. A 711 (2002) 27; Int. J. Mod. Physics A 18 (2003) 173.

[52] J. Dudek, et al., Eur. Phys. J. A48 (2012) 187. 